\title{
Life-threatening COVID-19 and aspergillosis co-infection in a heart transplant recipient: A cardiologist's nightmare
}

\author{
Renata Wachnicka-Truty ${ }^{1}$, Bartosz Curyłło ${ }^{2}$, Dagmara Wojtowicz ${ }^{1}$, \\ Dorota Kulawiak-Gałąska ${ }^{3}$, Marcin Renke ${ }^{4}, K_{\text {Katarzyna Sikorska }}$, \\ Marcin Gruchała ${ }^{2}$, Marek Koziński ${ }^{1}[0$ \\ ${ }^{1}$ Department of Cardiology and Internal Diseases, Institute of Maritime and Tropical Medicine, \\ Medical University of Gdansk, Gdynia, Poland \\ ${ }^{2}$ Department of Cardiology, Medical University of Gdansk, Gdansk, Poland \\ ${ }^{3}$ Department of Radiology, Medical University of Gdansk, Gdansk, Poland \\ ${ }^{4}$ Department of Occupational, Metabolic and Internal Diseases, \\ Institute of Maritime and Tropical Medicine, Medical University of Gdansk, Gdynia, Poland \\ ${ }^{5}$ Department of Tropical and Parasitic Diseases, Institute of Maritime and Tropical Medicine, \\ Medical University of Gdansk, Gdynia, Poland
}

The coronavirus disease 2019 (COVID-19) has become an unprecedented threat to solid-organ transplant (SOT) recipients, with excessive mortality observed in infected patients [1]. These immunocompromised patients with frequent comorbidities easily get COVID-19 and are at high risk of developing concomitant infections $[2,3]$. Additionally, their response to COVID-19 vaccine may be suboptimal [4]. At the early stage of the pandemic, the appropriate management of COVID-19-infected SOT recipients was unclear and even now is only based on expert recommendations $[5,6]$. Moreover, due to ongoing immunosuppression, some SOT recipients may remain positive for severe acute respiratory syndrome coronavirus-2 (SARS-CoV-2) for an extended period [7].

The goal herein, is to present diagnostic and therapeutic difficulties in a heart transplant recipient who was co-infected with COVID-19 and aspergillosis. We also briefly discuss the infection risk and optimal management of patients after heart transplantation in the setting of the COVID-19 pandemic.
A 59-year-old male presented and was admitted with COVID-19 to our department in October 2020. The patient underwent orthotropic heart transplantation due to dilated cardiomyopathy 5 months prior. The post-transplant course was complicated by invasive aspergillosis of the left lung which was diagnosed 3 months after heart transplantation. The patient presented with shortness of breath with mild exercise, slightly decreased pulse oximetry $\left(\mathrm{SpO}_{2}\right.$ 93-94\%), fever, mild sinus tachycardia, left hydrothorax and elevated C-reactive protein (CRP) concentration $(54 \mathrm{mg} / \mathrm{dL})$. The diagnosis of pulmonary aspergillosis was finally established based on the results of computed tomography (CT; Fig. 1A, B) and transthoracic biopsy, while bronchoalveolar lavage testing for Aspergillus galactomannan antigen was negative. In-hospital therapy with voriconazole was initiated. After a transient clinical improvement, the patient started to complain of sweatiness and recurrent fever, and his CRP and procalcitonine concentrations rose from 12 to $172 \mathrm{mg} / \mathrm{L}$ and from 0.05 to $0.59 \mathrm{ng} / \mathrm{mL}$, respectively. After two negative results, the third

Address for correspondence: Prof. Marek Koziński, MD, PhD, FESC, Department of Cardiology and Internal Diseases, Institute of Maritime and Tropical Medicine, Medical University of Gdansk, ul. Powstania Styczniowego 9B, 81-519 Gdynia, Poland, tel: +48 5869984 06, e-mail: marek.kozinski@gumed.edu.pl

\section{Received: 17.11.2021 Accepted: 13.12.2021 Early publication date: 11.01.2022}

This article is available in open access under Creative Common Attribution-Non-Commercial-No Derivatives 4.0 International (CC BY-NC-ND 4.0) license, allowing to download articles and share them with others as long as they credit the authors and the publisher, but without permission to change them in any way or use them commercially. 


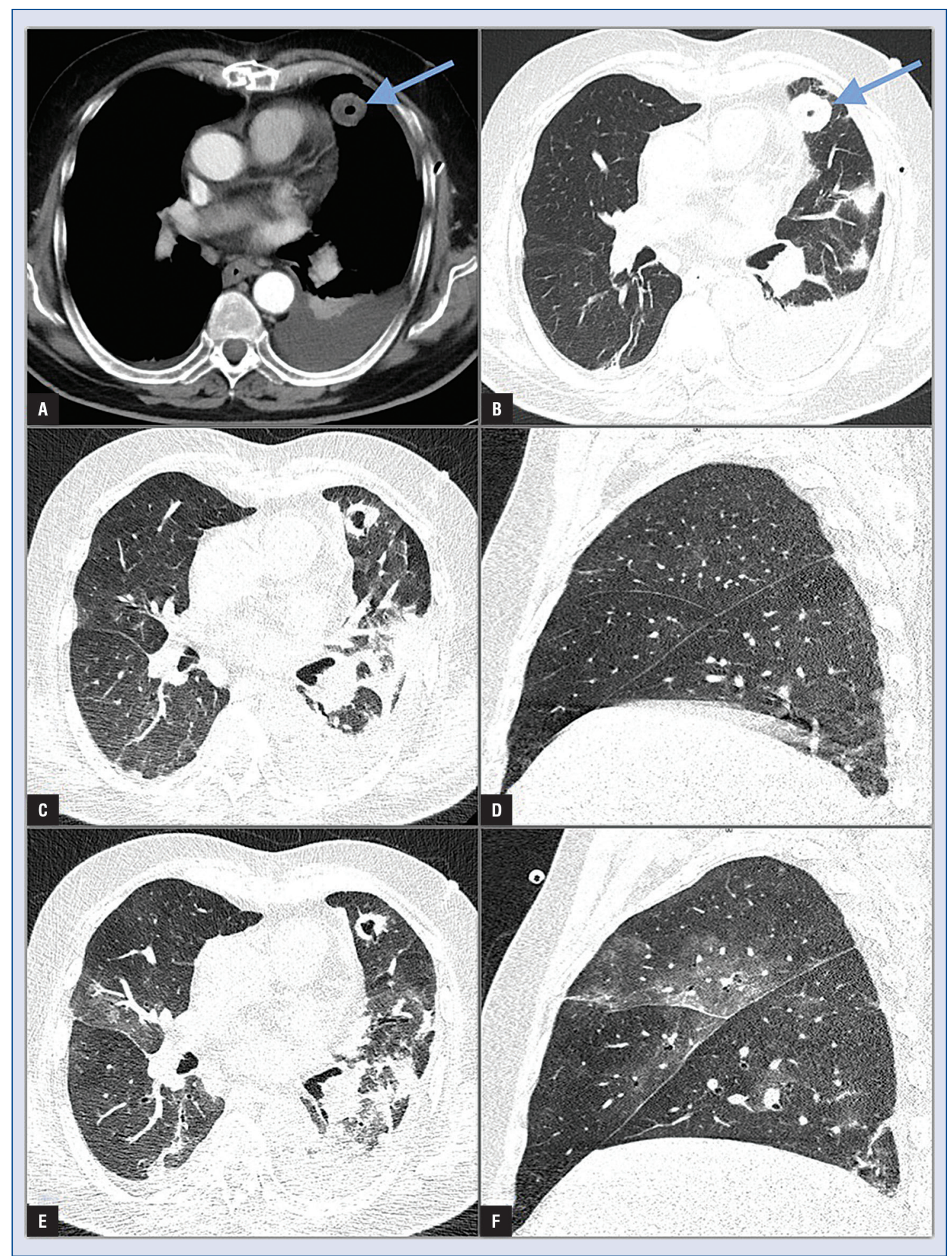

Figure 1. Evolution of pulmonary lesions. Invasive pulmonary aspergillosis with a cavitary lesion (blue arrows) diagnosed in a transplantation center (upper panel). Upper left: computed tomography (CT) scan (A; axial lung window). Upper right: post contrast CT scan (B; axial mediastinal widow). CT scans performed on admission to a coronavirus disease 2019 (COVID-19) unit indicating no evidence of viral pneumonia (middle panel). At this time, the patient already had a positive real-time polymerase chain reaction test for severe acute respiratory syndrome coronavirus- 2 . Middle left: axial lung window (C). Middle right: sagittal lung window (D). CT scans performed in a COVID-19 unit when the patient suffered from acute respiratory failure (lower panel). Subpleural areas of ground-glass opacity indicating COVID-19 pneumonia are present. Lower left: axial lung window (E). Lower right: sagittal lung window (F). 
real-time polymerase chain reaction (RT-PCR) test for SARS-CoV-2 was positive. Then the patient was transferred from a transplantation center to the cardiology department, which had been transformed into a unit dedicated for COVID-19 patients. On admission, the patient was in a relatively good condition with room-air oximetry of $94 \%$. In laboratory examinations, microcytic anemia with a hemoglobin concentration of $8.8 \mathrm{~g} / \mathrm{dL}$ and a mean corpuscular volume of $82 \mathrm{fL}$ as well as leucopenia $2.9 \mathrm{G} / \mathrm{L}$ was found. On admission, a chest $\mathrm{CT}$ was performed which revealed lesions typical for pulmonary aspergillosis, without any signs of COVID-19 pneumonia (Fig. 1C, D). Voriconazole was stopped and liposomal amphotericin B together with piperacillin and tazobactam were started. Subsequently, the immunosuppressive treatment was modified (i.e., mycophenolate mofetil was stopped and tacrolimus with prednisone was continued). CRP concentration decreased to $40 \mathrm{mg} / \mathrm{L}$. In the next days, the condition of the patient was substantially deteriorating. Dyspnea at rest, an increase in fever up to $39^{\circ} \mathrm{C}$ and low room-air oximetry $(83 \%)$ was observed. Leukocyte count dropped to 0.432 $\mathrm{G} / \mathrm{L}$, while hemoglobin concentration was at the level of $7.1 \mathrm{~g} / \mathrm{dL}$. CRP concentration rose do 250 $\mathrm{mg} / \mathrm{L}$ with borderline procalcitonin concentration of $0.40 \mathrm{ng} / \mathrm{mL}$. Supplemental oxygen therapy (up to $7 \mathrm{~L} / \mathrm{min}$ ) through a face mask was implemented. Staphylacoccus epidermidis, typically colonizing healthy human skin, was identified in two blood cultures. The central venous line was removed. Its tip was sent for a culture (which was negative) and another central venous line was inserted on the other side of the patient's body. Another chest CT demonstrated abnormalities characteristic for COVID-19 pneumonia with the extent of the involved lung tissue estimated to be $30 \%$ together with the co-existing pulmonary aspergillosis (Fig. 1E, F).

The management of this very complex patient was extensively discussed with numerous specialists (e.g., cardiologist familiar with transplantology, pulmonology, radiology, clinical microbiology, hematology and infectious disease specialists), both from the centers where the transplant was done and where he was then being treated. Apart from COVID-19 pneumonia and pulmonary aspergillosis, there was also suspicion of sepsis of mixed (bacterial and fungal) etiology. Additionally, poliethiological anemia (caused by iron deficiency, inflammation and myelosuppression) was diagnosed. The patient was treated with remdesivir, convalescent plasma, red blood cell transfusion, filgrastim, meropenem, teicoplanin, levofloxacin and prednisone was changed to dexamethasone. The tacrolimus dosage was adjusted several times according to its blood concentration. Intensive rehabilitation was implemented. A gradual recovery of the patient was witnessed. Three weeks after admission, respiratory support with supplemental oxygen was no longer necessary. CRP concentration dropped to $7 \mathrm{mg} / \mathrm{L}$, while the leukocyte count was at the level of $7.0 \mathrm{G} / \mathrm{L}$. A control chest CT showed regression of the COVID-19-related lesions with stable lesions attributed to pulmonary aspergillosis. On day 23 of hospitalization in the COVID-19 unit, a nasopharyngeal swab was collected and the RT-PCR test for SARS-CoV-2 had a negative result. The patient was then transferred back to the transplantation center in good condition where antifungal therapy was completed. Six months after his recovery from COVID-19 pneumonia the patient developed an asymptomatic cytomegalovirus infection which was successfully treated with ganciclovir. After recovery, the patient received two doses of the COVID-19 vaccine. At 12-month follow-up, the patient was doing well, without any limitation to everyday life, free from opportunistic infections.

COVID-19 associated pulmonary aspergillosis (CAPA) is a new phenomenon, recently discussed in the literature [8]. Contrary to the patient discussed here, in a CAPA setting, COVID-19 pneumonia typically precedes aspergillosis and a continuum of steps (respiratory colonization, tissue invasion and angioinvasion) are required for its development. Critically ill COVID-19 patients, including SOT recipients, are particularly prone to CAPA. In fact, cases of SOT recipients initially infected with aspergillosis and subsequently developing COVID-19 are rarely reported [9].

This case report illustrates how vulnerable to SARS-CoV-2 and other infections SOT recipients are and how challenging their treatment may be when COVID-19 pneumonia develops and it also shows the power of multidisciplinary cooperation. We strongly believe that in order to prevent life-threatening COVID-19 pneumonia, patients after heart transplantation should receive a timely vaccination, including a booster dose [10]. Other key interventions in this population are: screening for opportunistic pathogens and effective prevention of community and hospital acquired infections.

Conflict of interest: None declared 


\section{References}

1. Jering KS, McGrath MM, Mc Causland FR, et al. Excess mortality in solid organ transplant recipients hospitalized with COVID-19: A large-scale comparison of SOT recipients hospitalized with or without COVID-19. Clin Transplant. 2022; 36(1): e14492, doi: 10.1111/ctr.14492, indexed in Pubmed: 34558116.

2. Skonieczny P, Heleniak Z, Szostakiewicz M, et al. Coinfection of COVID-19 and pneumocystosis in a patient after kidney transplantation. Pol Arch Intern Med. 2021; 131(6): 566-567, doi: 10.20452/pamw.15996, indexed in Pubmed: 33973748.

3. Kalil AC, Florescu DF. Mortality in solid organ transplant recipients hospitalized for COVID-19. Am J Transplant. 2022; 22(1): 12-13, doi: 10.1111/ajt.16885, indexed in Pubmed: 34738312.

4. Boyarsky BJ, Werbel WA, Avery RK, et al. Antibody response to 2-dose SARS-CoV-2 mRNA vaccine series in solid organ transplant recipients. JAMA. 2021; 325(21): 2204-2206, doi: 10.1001/ jama.2021.7489, indexed in Pubmed: 33950155.

5. Ilonze OJ, Ballut K, Rao RS, et al. SARS-CoV-2 infection in heart transplant recipients: a systematic literature review of clinical outcomes and immunosuppression strategies. Heart Fail Rev. 2021 [Epub ahead of print], doi: 10.1007/s10741-021-10181-y, indexed in Pubmed: 34671872.
6. Limaye AP, Hardinger K. COVID-19: Issues related to solid organ transplantation. In: the UpToDate website. https://www. uptodate.com/contents/covid-19-issues-related-to-solid-organ-transplantation (Accessed on 11.11.2021).

7. Benotmane I, Risch S, Doderer-Lang C, et al. Long-term shedding of viable SARS-CoV-2 in kidney transplant recipients with COVID-19. Am J Transplant. 2021; 21(8): 2871-2875, doi: 10.1111/ajt.16636, indexed in Pubmed: 33961334.

8. Verweij PE, Brüggemann RJM, Azoulay E, et al. Taskforce report on the diagnosis and clinical management of COVID-19 associated pulmonary aspergillosis. Intensive Care Med. 2021; 47(8): 819-834, doi: 10.1007/s00134-021-06449-4, indexed in Pubmed: 34160631.

9. Vidanapathirana M, Minuvanpitiya G, Karunaratne R, et al. Triple infection with disseminated tuberculosis, invasive aspergillosis and COVID-19 in an organ transplant recipient with iatrogenic immunosuppression. BMJ Case Rep. 2021; 14(8), doi: 10.1136/bcr-2021-245131, indexed in Pubmed: 34376424.

10. Rosano G, Jankowska EA, Ray R, et al. COVID-19 vaccination in patients with heart failure: a position paper of the Heart Failure Association of the European Society of Cardiology. Eur J Heart Fail. 2021; 23(11): 1806-1818, doi: 10.1002/ejhf.2356, indexed in Pubmed: 34612556. 